\title{
Prevalence De L'insuffisance Cardiaque Au Cours De La Grossesse Et Dans Les Suites De Couches En Milieu Hospitalier A Parakou En 2015
}

\author{
Codjo Léopold Houétondji \\ Maître Assistant-CAMES \\ Unité de Cardiologie, Faculté de Médecine, Université de Parakou, Bénin \\ Adjagba Philippe Mahougnon \\ Assistant Chef de Clinique-CAMES \\ Sonou Jijoho Arnaud \\ Assistant Chef de Clinique-CAMES \\ Hounkponou Murielle \\ Assistant Chef de Clinique-CAMES \\ Unité de Soins d'Enseignement et de Recherche en Cardiologie, Faculté de \\ Sciences de la Santé, Université d'Abomey Calavi, Cotonou, Bénin

\section{Hounkponou Fanny} \\ Maître Assistant-CAMES \\ Unité de Gynécologie et d'Obstétrique, Faculté de Médecine, \\ Université de Parakou, Bénin \\ Dohou Serge Hugues Mahougnon, MD \\ Djoh Khadidja Ingrid, MD \\ Alassani Adébayo, MD \\ Ahoui Séraphin \\ Assistant Chef de Clinique-CAMES \\ Service de Médecine, Centre Hospitalier Universitaire Départemental \\ Borgou, Parakou, Bénin

\section{Houénassi Dèdonougbo Martin} \\ Professeur Titulaire-CAMES \\ Unité de Soins d'Enseignement et de Recherche en Cardiologie, Faculté de \\ Sciences de la Santé, Université d'Abomey Calavi, Cotonou, Bénin
}

Doi: 10.19044/esj.2018.v14n6p201 URL:http://dx.doi.org/10.19044/esj.2018.v14n6p201

Abstract

Introduction: Heart failure is one of peripartum complications which contribute to maternal mortality. But the epidemiological features of this disease are not well known in Benin. This paper aims at studying the 
prevalence and associated factors of heart failure during pregnancy and the postpartum in Parakou in 2015. Methods: It was a descriptive and analytic cross sectional study scheduled from March to July 2015. We recruited systematically all pregnant or parturient women, met in Parakou's hospitals. The inclusion criteria were the presence of at least one cardiovascular symptom. Diagnosis of heart failure was made by using the guidelines of the European Society of Cardiology and of the American Society of Echocardiography. Results: During the survey, 2503 pregnant or parturient women were admitted and $25(0,99 \%)$ were diagnosed with heart failure. Dyspnea was the main symptom (96\%). Heart failure was caused by peripartum cardiomyopathy $(84 \%)$, hypertensive cardiomyopathy (12\%) and rheumatic heart disease $(4 \%)$. Heart failure was more prevalent in patients with the following conditions: age $\geq 30$ years $(p=0,001)$, low socioeconomic level $(p=0,02)$, multigestational status $(p=0,002)$ and multiparity $(p<0,001)$. The management of heart failure was classic. The main difficulty noted was financial problems with $36 \%$ of patients unable to afford the treatment. No case of death was counted during the study. Conclusion: In Parakou, heart failure occurs mostly during the post partum period. Dyspnea was the main symptom. The main etiology was peripartum cardiomyopathy. The associated conditions were age $\geq 30$ years, low socioeconomic level, multigestational status and multiparity.

Keywords: Peripartum cardiomyopathy - pregnancy - heart failure - Africa

\section{Résumé}

Introduction : L'insuffisance cardiaque est l'une des complications graves de la période du péripartum. Ce travail vise à déterminer la prévalence de l'insuffisance cardiaque (IC) et les facteurs associés au cours de la grossesse et dans les suites de couches en milieu hospitalier à Parakou en 2015. Méthodes : Il s'agit d'une étude transversale descriptive et analytique réalisée du 01 Mars 2015 au 31 Juillet 2015. Les parturientes et gestantes suivies en consultation ou hospitalisées dans les hôpitaux de Parakou, présentant au moins un signe d'appel cardiovasculaire ont été systématiquement recensées. Le diagnostic d'IC a été fait selon les recommandations de la Société Européenne de Cardiologie et de la Société Américaine d'Echocardiographie. Résultats : Sur les 2503 gestantes et parturientes admises durant la période, $25(0,99 \%)$ avaient une IC. L'âge moyen était de 28,55 $\pm 6,86$ ans. Tous les cas d'IC sont survenus en post partum. Le principal symptôme était la dyspnée (96\%). Les différentes causes d'IC retrouvées étaient : la cardiomyopathie du péripartum (84\%), la cardiomyopathie hypertensive (12\%) et l'insuffisance mitrale d'origine rhumatismale $(4 \%)$. Les facteurs associés à l'insuffisance cardiaque étaient l'âge $\geq 30$ ans, le bas niveau socioéconomique, la multigestité 
et la multiparité. Le traitement de l'IC a été classique mais 36\% des patientes ne pouvaient se l'offrir. Néanmoins, aucun décès n'a été recensé. Conclusion : A Parakou en 2015, l'insuffisance cardiaque associée à la grossesse est essentiellement survenue dans le post partum. La dyspnée était le principal symptôme et la cardiomyopathie du péripartum, la principale cause.

Mots Clés: Cardiomyopathie, péripartum, grossesse, Insuffisance cardiaque, Afrique Sub-saharienne

\section{Introduction}

La période du péripartum et des suites de couches est dominée par des complications graves. On peut citer l'éclampsie, l'hématome rétro placentaire, l'hémorragie de la délivrance, l'embolie pulmonaire [Schellpfeffer et al, 2015 ; Lutomski et al, 2011]. Habituellement moins bruyante et relativement peu fréquente, l'insuffisance cardiaque vient compléter la liste de ces complications [Denis, 2015 ; Ntusi et al, 2015]. Elle constitue une affection grave car responsable d'une forte mortalité chez les victimes [Ntusi et al, 2015 ; Arany et al, 2016]. Les manifestations cliniques ne sont pas spécifiques et le début est plutôt insidieux avec des signes qui se confondent presque avec les modifications physiologiques de la grossesse [Goland et al, 2009; Groesdonk et al, 2009]. Dans les pays développés, les causes de l'insuffisance cardiaque du péripartum sont dominées par les cardiopathies congénitales opérées [Van Mook et al, 2005]. Dans ces pays, un antécédent documenté de chirurgie cardiaque ou de cardiopathie congénitale est donc important à rechercher et attire l'attention des obstétriciens, imposant ainsi une surveillance minutieuse de l'état cardiovasculaire des gestantes. En Afrique, quelques facteurs favorisant l'insuffisance cardiaque dans le péripartum ont été identifiés particulièrement par certaines études rétrospectives. Il s'agit de l'anémie, la malnutrition et la précarité [Mongo Ngamami et al, 2004; Katibi, 2003]. Au nord du Bénin, la pratique clinique quotidienne semble révéler une fréquence assez élevée de l'insuffisance cardiaque du post-partum. Cette étude a été donc initiée pour évaluer la prévalence, les manifestations cliniques et les facteurs associés à l'insuffisance cardiaque du péripartum et des suites de couches en milieu hospitalier à Parakou en 2015.

\section{Méthodes}

L'étude était transversale descriptive et analytique avec collecte prospective des données. Elle s'est déroulée du 01 Mars au 31 Juillet 2015 dans les services de gynéco-obstétrique et de cardiologie de l'Hôpital d'Instruction des Armées de Parakou et du Centre Hospitalo-Universitaire et Départemental du Borgou. 
Le recrutement était systématique et a concerné toutes les gestantes, parturientes et femmes dans les six premiers mois de suite de couches ayant présenté l'un au moins des signes ci-après : une dyspnée à partir du stade II de la NYHA, des œdèmes des membres inferieurs bilatéraux, des palpitations ou une asthénie à l'effort. Ont été exclues de notre étude, les patientes qui n'avaient aucun signe d'insuffisance cardiaque et/ou qui avaient un échoDoppler cardiaque normal ou non réalisée.

Une fiche d'enquête préétablie a été utilisée pour le recueil des données sociodémographiques, les facteurs de risque cardiovasculaire, les antécédents personnels et familiaux de maladies cardiovasculaires, le suivi de la grossesse et les données thérapeutiques et évolutives. L'appréciation du niveau économique était basée sur la qualité du domicile, la profession, le revenu mensuel et le moyen de déplacement comme nous l'avions décrit précédemment [Codjo et al, 2015].

L'enquête s'est déroulée en 3 étapes: la présélection des patientes à partir des signes d'appel cardiovasculaire par les consultants; l'examen clinique réalisé par l'enquêteur. L'électrocardiogramme et l'échoDoppler cardiaque, de même que la synthèse diagnostique pour chaque patiente ont été réalisés par un même cardiologue. L'évaluation des fonctions ventriculaires et des différentes valvulopathies a été faite selon les recommandations 2011 de la Société Américaine d'Echocardiographie [ASE, 2011]. Les diagnostics positif et étiologique de l'insuffisance cardiaque au cours de la grossesse ont été faits conformément aux normes de la société européenne de cardiologie [Regitz-Zagrosek et al, 2011 ; McMurray et al, 2012]. Ainsi, la dysfonction systolique du ventricule gauche a été retenue devant une fraction d'éjection (FE) $<50 \%$ et/ou une fraction de raccourcissement (FR) $<25 \%$. Les pressions de remplissage ventriculaire, expression de la fonction diastolique, ont été évaluées sur la base de l'analyse du flux transmitral (Doppler pulsé et tissulaire), de la FE et de la taille de l'oreillette gauche. Les critères diagnostiques de la cardiomyopathie du péripartum (CMPP) étaient : - la survenue d'une insuffisance cardiaque congestive dans le mois précédent ou les 5 mois suivant l'accouchement; - l'absence de cause retrouvée à la cardiomyopathie malgré un bilan étiologique; - l'absence de cardiopathie connue avant l'apparition des premiers symptômes ; - la présence de signes échocardiographiques attestant la dysfonction ventriculaire gauche; une FEVG $<45 \%$ et/ou une fraction de raccourcissement $<30 \%$ et/ou une dilatation cavitaire avec un diamètre télédiastolique ventriculaire $>2,7 \mathrm{~cm} / \mathrm{m} 2$ de surface [Regitz-Zagrosek et al, 2011]. La myocardiopathie hypertensive a été retenue devant l'antécédent d'hypertension artérielle et une masse ventriculaire gauche à l'échoDöppler cardiaque élevée (supérieure à $115 \mathrm{~g} / \mathrm{m}^{2}$ chez l'homme et $95 \mathrm{~g} / \mathrm{m}^{2}$ chez la femme) [ASE, 2011]. La valvulopathie rhumatismale chronique a retenue devant l'épaississement valvulaire associé 
ou non à une fusion commissurale avec ou sans remaniement de l'appareil sous valvulaire [ASE, 2011].

Les données ont été analysées à l'aide du logiciel Epi info version 7. L'association entre les variables est déterminée à l'aide du test de Chi carré de Pearson au seuil de significativité de $\mathrm{p}<0,05$.

\section{Résultats}

\section{- $\quad$ Prévalence de l'insuffisance cardiaque}

Sur les 2503 patientes admises dans les différents services durant la période d'étude, 180 avaient présenté une affection cardiovasculaire dont 25 cas d'insuffisance cardiaque soit une prévalence de 0,99\% (figure 1).

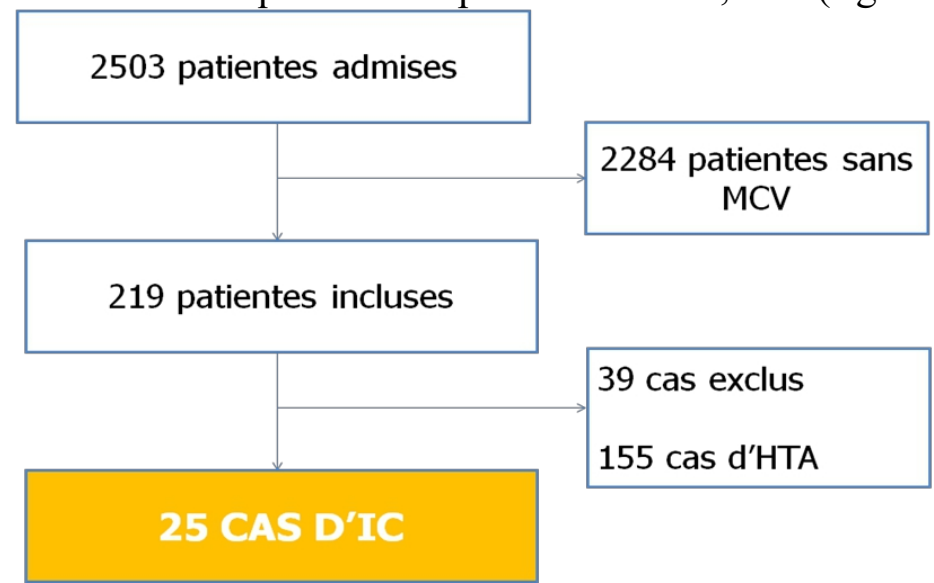

Figure 1 : Diagramme de flux montrant la sélection des parturientes victimes d'insuffisance cardiaque à Parakou en 2015

\section{- $\quad$ Caractéristiques générales des patientes}

L'âge moyen des patientes était de 28,55 $\pm 6,86$ ans avec des extrêmes de 16 et 45 ans. Les femmes victimes d'insuffisance cardiaque n'étaient pas scolarisées dans $48 \%$ des cas et le niveau socioéconomique était bas chez 20(80\%) d'entre elles. Ces données sont présentées dans le tableau 1.

Tableau 1 : Caractéristiques générales des gestantes, parturientes et femmes en suites de couches victimes d'insuffisance cardiaque à Parakou en $2015(\mathrm{n}=25)$.

\begin{tabular}{ccc}
\hline & Effectifs & Pourcentage (\%) \\
\hline Age & & 36 \\
$\leq 30$ ans & 9 & 64 \\
$>30$ ans & 16 & \\
Gestité & & 20 \\
$\leq 3$ & 5 & 80 \\
$>3$ & 20 & 24 \\
Parité & & 76 \\
$\leq 3$ & 6 &
\end{tabular}

Situation matrimoniale 


\begin{tabular}{ccc}
\hline En couple & 21 & 84 \\
Célibataire & 4 & 16 \\
Niveau de scolarisation & & 48 \\
Non scolarisé & 12 & 40 \\
Primaire/ secondaire & 10 & 12 \\
Supérieur & 3 & 80 \\
Niveau socio-économique & & 16 \\
Bas & 20 & 4 \\
Moyen & 4 & \\
Elevé & 1 & \\
\hline
\end{tabular}

\section{Caractéristiques cliniques, électrocardiographiques et échocardiographiques des patientes (tableau 2)}

La totalité des patientes en insuffisance cardiaque était en post partum. Les symptômes apparaissaient surtout au cours des trois premiers mois (84\% des cas). La dyspnée était le signe fonctionnel le plus retrouvé (24 patientes soit 96\%). Selon la classification de la New York Heart Association (NHYA), 11 patientes $(45,83 \%)$ étaient au stade IV, $07(29,17 \%)$ au stade III et 06 (25\%) au stade II. Une tachycardie était observée chez 21 patientes (84\%) et un souffle d'insuffisance mitrale chez 10 autres (40\%). La décompensation était globale chez $72 \%$ des patientes.

A l'électrocardiogramme, le rythme était sinusal mais une tachycardie sinusale et une hypertrophie ventriculaire gauche ont été notées chez 16 patientes (64\%). L'aspect échographique le plus observé était une dilatation ventriculaire gauche $(76 \%)$ associée à une dilatation biatriale $(48 \%)$ et une insuffisance mitrale fonctionnelle (68\%). On notait une dysfonction systolique dans la plupart des cas (84\%) avec une élévation des pressions de remplissage (profil mitral normalisé dans $48 \%$ et veine cave inférieure dilatée dans $72 \%$ ). Il a été noté, chez une patiente de 30 ans, une maladie mitrale moyennement sévère sans retentissement hémodynamique significatif (gradient transmitral à $6 \mathrm{mmHg}$ ). L'origine rhumatismale a été retenue devant les anomalies échographiques suivantes: valves épaissies remaniées, discrète fusion commissurale, cordages rétractées et épaissies.

Les différentes causes d'insuffisance cardiaque retrouvées dans notre étude étaient: la cardiomyopathie du péripartum (CMPP) à $84 \%$, la cardiomyopathie hypertensive à $12 \%$ et la maladie mitrale d'origine rhumatismale à $4 \%$.

Tableau 2 : Caractéristiques cliniques, électrocardiographiques et échocardiographiques des gestantes, parturientes et femmes en suites de couches victimes d'insuffisance cardiaque à

Parakou en $2015(n=25)$

\begin{tabular}{ccc}
\hline & Effectifs & Pourcentage (\%) \\
\hline Signes cliniques & & \\
Dyspnée d'effort & 24 & 96 \\
Palpitations & 3 & 12 \\
\hline
\end{tabular}




\begin{tabular}{|c|c|c|}
\hline Hépatalgie d'effort & 11 & 44 \\
\hline Toux & 17 & 68 \\
\hline Edèmes & 14 & 56 \\
\hline Tachycardie & 21 & 84 \\
\hline Souffle cardiaque d'insuffisance mitrale & 10 & 40 \\
\hline \multicolumn{3}{|l|}{ Syndromes } \\
\hline Insuffisance cardiaque gauche & 7 & 28 \\
\hline Insuffisance cardiaque droite & 0 & 0 \\
\hline Insuffisance cardiaque globale & 18 & 72 \\
\hline Anémique & 3 & 12 \\
\hline Réponse inflammatoire systémique & 3 & 12 \\
\hline \multicolumn{3}{|l|}{ Anomalies électrocardiographiques } \\
\hline Tachycardie & 16 & 64 \\
\hline Déviation axiale gauche & 4 & 16 \\
\hline Déviation axiale droite & 0 & 0 \\
\hline Surcharge ventriculaire gauche & 16 & 64 \\
\hline Surcharge atriale gauche & 6 & 24 \\
\hline Surcharge cavitaire droite & 0 & 0 \\
\hline Troubles de la repolarisation & 14 & 56 \\
\hline \multicolumn{3}{|l|}{ Anomalies échographiques } \\
\hline Dilatation ventriculaire & 19 & 76 \\
\hline Dilatation auriculaire & 15 & 60 \\
\hline Hypertrophie VG & 8 & 32 \\
\hline $\mathrm{FEVG}^{*}<45 \%$ & 21 & 84 \\
\hline $\mathrm{FEVG} \geq 45 \%$ & 4 & 16 \\
\hline Dysfonction diastolique du VG & 18 & 72 \\
\hline $\mathrm{TAPSE}^{\circ}<15 \mathrm{~mm}$ & 5 & 20 \\
\hline HTAP (PAPS $\geq 50 \mathrm{mmHg}$ ) & 9 & 36 \\
\hline $\begin{array}{l}\text { Anomalies valvulaires organiques } \\
\text { (Maladie mitrale) }\end{array}$ & 1 & 4 \\
\hline
\end{tabular}

*Fraction d'Ejection Ventriculaire Gauche

${ }^{\circ}$ Tricuspid Annular Plane Systolic Excursion

\section{- $\quad$ Facteurs associés à l'insuffisance cardiaque}

Les facteurs de risque significativement associés à l'insuffisance cardiaque dans notre enquête étaient l'âge $\geq 30$ ans $(p<0,001)$, l'absence de scolarisation $(\mathrm{p}=0,02)$, le bas niveau socioéconomique $(\mathrm{p}<0,02)$, la multigestité $(\mathrm{p}<0,002)$ et la multiparité $(\mathrm{p}<0,001)$ (Tableau 3$)$.

Tableau 3: Facteurs associés à l'insuffisance du post-partum à Parakou en $2015(\mathrm{n}=25)$

$\begin{array}{lccc}\text { Variables } & \text { Total } & \begin{array}{c}\text { Insuffisance } \\ \text { cardiaque }\end{array} & \text { RP[IC à 95\%] }\end{array}$

\begin{tabular}{ccccc}
\hline Age & & & $1,92[1,05-4,55]$ & 0,001 \\
$\leq 30$ ans & 69 & 9 & & \\
>30ans & 111 & 16 & $2,42[1,10-5,33]$ & 0,02 \\
Niveau d'instruction & & & & \\
Non scolarisé & 84 & 12 & & \\
Scolarisé & 96 & 8 & & \\
\hline
\end{tabular}




\begin{tabular}{ccccc}
\hline Niveau socioéconomique & & & & \\
Bas & & & $6,42[0,89-45,96]$ & 0,02 \\
Non bas & 142 & 20 & & \\
Obésité & 38 & 5 & $0,42[0,06-2,95]$ & 0,35 \\
Oui & 16 & 1 & & \\
Non & 164 & 24 & & 0,0002 \\
Gestité & 108 & 5 & $3,40[1,88-5,08]$ & \\
$\leq 3$ & 72 & 20 & & $10^{-7}$ \\
$>3$ & 132 & 6 & $2,90[201-4,77]$ & \\
Parité & 48 & 19 & & 0,608 \\
$\leq 3$ & 166 & 23 & $0,96[0,80-1,25]$ & \\
$>3$ & 14 & 2 & & 0,564 \\
Type de grossesse & & & $1,11[0,29-4,22]$ & \\
Monofoetale & 13 & 2 & & \\
Gémellaire & 167 & 23 & & \\
Toxémie gravidique & & & & \\
Oui & & & \\
Non & & & & \\
\hline
\end{tabular}

\section{Traitement et évolution}

Le traitement a été médicamenteux associant des diurétiques, des inhibiteurs de l'enzyme de conversion et des bêtabloquants. Les inhibiteurs calciques (amlodipine) avaient été associés chez les patientes ayant une hypertension artérielle. L'allaitement maternel a été systématiquement proscrit chez toutes les patientes. La principale difficulté retrouvée dans la prise en charge était d'ordre financier. En effet, 36\% des patientes ne pouvaient pas acheter le traitement prescrit. Aucun décès n'a été recensé au cours de notre enquête.

\section{Discussion}

Notre objectif était de déterminer la prévalence de l'insuffisance cardiaque, ses étiologies et les facteurs associés chez les gestantes, les parturientes et les femmes en suites de couches suivies en milieu hospitalier à Parakou. La prise en compte de tous les centres hospitaliers de la ville de Parakou, le recrutement systématique de toutes les gestantes et l'examen systématique de toutes ces dernières a permis d'identifier tous les cas d'insuffisance cardiaque en milieu hospitalier durant la période notre étude. L'évaluation clinique et échographique de ces dernières par un cardiologue garantit la fiabilité du diagnostic.

La principale limite de ce travail réside dans la stratégie diagnostique de l'insuffisance cardiaque chez nos patientes. En effet, des patientes qui avaient des signes non spécifiques (dyspnée, œdème, asthénie et/ou tachycardie) mais dont l'échoDoppler cardiaque était normal avaient été exclues. Chez ces dernières, le dosage de BNP ou du NT-proBNP aurait pu 
aider à améliorer le diagnostic d'insuffisance cardiaque. Ce test très bénéfique a une haute sensibilité et donc une excellente valeur prédictive négative d'exclusion du diagnostic d'insuffisance cardiaque chez les patients symptomatiques. Ainsi une concentration de NT-proBNP < $300 \mathrm{ng} / \mathrm{L}$ aurait permis d'exclure le diagnostic d'insuffisance cardiaque avec une valeur prédictive négative de $98 \%$ [Jourdain et al, 2009]. Malheureusement, ce test n'a pu être réalisé du fait d'une inaccessibilité technique dans notre région. La stratégie d'exclusion systématique de ces patientes symptomatiques a probablement favorisé une sous estimation de la prévalence de l'insuffisance cardiaque dans notre étude. Malgré cette insuffisance, les données de cette étude seront très utiles pour les stratégies de dépistage et de prise en charge de l'insuffisance cardiaque de même pour les études ultérieures.

La prévalence hospitalière de l'insuffisance cardiaque dans les suites de couches dans notre étude était de 0,99\%. Aux Etats-Unis en 2004, Klein et al ont rapporté une incidence stable des cardiopathies aigues sur grossesse entre 0,1 et $1,40 \%$ [Klein et al, 2004]. Steer a retrouvé $1 \%$ aux Royaume-Unis en 2005 [Steer, 2005], contre 0,28\% de Khemiri en Tunisie en 2015 [Khemiri et al, 2015]. Ces taux apparemment faibles ne sont pas à négliger compte tenu de la gravité des affections cardiovasculaires. A Parakou comme dans la plupart des pays pauvres, le suivi prénatal est assuré par les paramédicaux qui ont une mauvaise connaissance de ces affections cardiovasculaires [Codjo et al, 2014]. Des techniques simples de dépistage des maladies cardiovasculaires doivent être enseignées à ces soignants de premières lignes qui jouent un rôle important dans les systèmes sanitaires de notre pays.

Dans notre étude, tous les cas d'insuffisance cardiaque ont été observés dans le post-partum. Ce constat se rapproche des résultats de Machihude et al au Togo en 2012 [Machihude et al, 2014] et de Zabsonre et al au Burkina en 2000 [Zabsonre et al, 2000] qui ont retrouvé respectivement 90,24\% et $91 \%$ de patientes victimes d'insuffisance cardiaque en post partum. De même, Elkayam et al [Elkayam et al, 2001] avaient noté que la majorité des patientes (84\%) avait présenté les symptômes au cours du premier mois suivant l'accouchement. En effet, les modifications hémodynamiques qui accompagnent la grossesse (accroissement du volume plasmatique et des besoins métaboliques, anémie relative, altération des résistances vasculaires périphériques, dilatation ventriculaire et accroissement du débit cardiaque) sont à l'origine de décompensation cardiaque dans le péripartum de cardiopathies préexistantes ou autonomes [Davido et al, 1990]. La dyspnée d'effort était le maitre symptôme et elle était sévère chez la plupart des patientes (75\% au stade III ou IV NYHA). Les mêmes observations ont été faites dans la littérature africaine [Machihude et al, 2014 ; Zabsonre et al, 2000]. Par contre, Tara et al en Iran avaient rapporté seulement 2\% de patientes au stade IV [Tara et al, 2015]. L'anémie était présente chez 12\% de 
nos patientes comparable aux 28,6\% retrouvés par Mongo et al au Congo [Mongo Ngamami et al, 2004]. L'anémie est l'une des anomalies non spécifiques pouvant être rencontrées au cours de la grossesse et qui contribue à la majoration des signes d'insuffisance cardiaque [Bahloul et al, 2009].

La cardiopathie sous jacente était la CMPP dans $84 \%$ des cas d'insuffisance cardiaque soit une prévalence de $0,83 \%$ des patientes ayant présenté une pathologie cardiovasculaire au cours de la grossesse et dans les suites de couches à Parakou. Ce taux est plus élevé que les données Sud Africaines (0,10\% en 1996)[ Cloatre et al, 1996] Chinoises (0,29\% en 2009) [Pearl, 2009], américaines (1/4025) et européennes (1/4075) [Brar et al, 2007]. En ce qui concerne la répartition de la CMPP selon la race, Brar et al avaient noté une prévalence 2,9 plus élevée chez les patientes noires américaines que celles de race blanche et 7 fois plus que les hispaniques. Ces différents résultats confirment que la race noire est également un facteur prédisposant à la CMPP [Brar et al, 2007]. Le taux de valvulopathie rhumatismale (4\%) retrouvé dans notre série est inférieur à ceux de Zabsonre (12\%) au Burkina [Zabsonre et al, 2000] et de Khemiri (62,50\%) en Tunisie [Khemiri et al, 2015]. De même, aucune cardiopathie congénitale n'a été enregistrée dans notre étude contrairement aux taux élevés (70-80\%) retrouvés dans les pays développés. Cette faible représentation des cardiopathies rhumatismales et congénitales dans les étiologies d'insuffisance cardiaque dans nos pays en voie développement peut s'expliquer par le décès des patientes avant l'âge de procréation. En effet, les travaux de Chaabouni et al à Sfax (Tunisie) [Chaabouni et al, 1999] et de Ngouala à Louga (Sénégal) [Ngouala et al, 2015] ont rapporté un faible accès à la chirurgie des cardiopathies infantiles et le fort taux de décès des enfants porteurs de cardiopathie infantiles non opérées. Ceci dénote du caractère sévère de ces cardiopathies et de l'insuffisance de leur prise en charge dans nos pays pauvres [Faivre et al, 2009].

Dans notre étude, l'âge $\geq 30$ ans était significativement associé à un taux plus élevé d'insuffisance cardiaque comme l'ont remarqué Mielniczuk et al [Mielniczuk et al, 2006], Machihude et al [Machihude et al, 2014]. De même, les patientes non scolarisées ont développé 2,42 fois plus d'insuffisance cardiaque que celles scolarisées. Les patientes avec un bas niveau socioéconomique étaient 6,42 fois plus atteintes que celles de niveau socio économique moyen ou élevé. Ces résultats sont comparables à ceux de Katibi au Nigeria [Katibi, 2003] et Mongo au Congo [Mongo Ngamami et al, 2004]. En réalité, les femmes en Afrique noire sont soumises à des travaux physiques intenses, à un climat chaud et humide et à une malnutrition (carence en vitamines et en micronutriments) qui constituent des terrains favorisants pour la survenue d'une CMPP. Aucune relation statistiquement significative n'a été remarquée entre l'insuffisance cardiaque du post partum et les facteurs de risque cardiovasculaire spécifiquement l'obésité. La gestité et la parité 
étaient significativement associées à l'insuffisance cardiaque dans notre enquête. Ainsi la multigestité (80\%) et la multiparité (76\%) peuvent donc être classées comme des facteurs de risques de la CMPP en accord avec les données internationales [Arany et al, 2016; Mongo Ngamami et al, 2004; Machihude et al, 2014]. Ce constat serait lié à l'accumulation des modifications cardiaques (anatomiques et fonctionnelles) en rapport avec chacune des gestations chez les multigestes et multipares [Ladouceur et al, 2011]. Nous n'avons pas observé d'association entre la toxémie gravidique et la CMPP. Cette absence d'association entre la toxémie gravidique et la CMPP a été aussi rapporté par d'autres travaux africains [Machihude et al, 2014 ; Mongo Ngamami et al, 2004] contrairement à ceux de la littérature européenne [Ladouceur et al, 2011; Shah et al, 2013].

\section{Conclusion}

A Parakou en 2015, la prévalence de l'insuffisance cardiaque était survenue essentiellement dans le post partum et sa prévalence était aussi élevée que celles retrouvées dans la littérature internationale. La dyspnée était le principal symptôme et la cardiomyopathie du péripartum, sa principale cause. Les facteurs associés à l'insuffisance cardiaque du post partum étaient la multiparité, la multigestité, l'âge de gestante $\geq 30$ ans, la précarité et le bas niveau d'instruction. Des études intégrant le dosage des peptides natriurétiques dans la stratégie diagnostique et portant un échantillon plus large sont nécessaires pour confirmer ces données.

Néanmoins, l'apparition dans le péripartum ou dans les suites de couches d'une dyspnée et/ou des œè̀mes périphériques doit conduire à la recherche systématique des signes d'insuffisance cardiaque.

\section{References:}

1. Arany Z, Elkayam U. Peripartum Cardiomyopathy. Circulation. 2016;133:1397-1409.

DOI: 10.1161/CIRCULATIONAHA.115.020491.

2. Bahloul M, Ben Ahmed MN, Laaroussi L, Chtara K, Kallel H, Dammak $\mathrm{H}$ et al. Myocardiopathie du péripartum: incidence, physiopathologie, manifestations cliniques, prise en charge thérapeutique et pronostic. Ann Franc Anes Réa 2009; 28 : 44-60.

3. Brar SS, Khan SS, Sandhu GK, Jorgensen MB, Parikh N, Hsu JW et al. Incidence, mortality and racial differences in péripartum cardiomyopathy. Am J Cardiol. 2007; 100(2):302-4.

4. Chaabouni M, Kamoun T, Mekki N, Mahfoudh A, Karray A, Daoud $\mathrm{M}$ et al. Aspects épidémiologiques et évolutifs des cardiopathies congénitales dans le service de pédiatrie de Sfax : A propos de 123 cas. Tunisie Médicale. 1999 ; 77(5) : 264-71 
5. Cloatre G, Gueye PM, Niang B, Haudrechy D, Wade B, Sane M et al. Particularités étiopathogéniques, échographiques et évolutives de la myocardiographie du post-partum. Med Trop. 1996; 56: 376-80.

6. Codjo HL, Attinsounon CA, Dovonou CA, Foundohou E, Dohou SHM, Houénassi DM. Prévalence des cardiopathies rhumatismales chez les jeunes collégiens a Parakou en 2012 (Bénin). ReMIM. 2015 ; $6: 38-46$

7. Codjo HL, Tossou M, Dohou H, Ahoui S, Dovonou CA, Adoukounou TA, Houenassi DM. Facteurs associes a l'observance thérapeutique chez les patients suivis pour hypertension artérielle à Parakou, Bénin. Annales de l'Université de Parakou, Série "Sciences de la Santé », 2014 ; 4 (1) : 10-3

8. Davido A, Artigou Jy, Chapelon C, Michel P L. Maladies cardiovasculaires et grossesse. Encycl. Méd. -Chir. $1990 ; 12: 1-12$.

9. Dennis AT. Heart failure in pregnant women: is it peripartum cardiomyopathy? Anesth Analg. 2015;120(3):638-43. doi: 10.1213/ANE.0000000000000597.

10. Elkayam U, Tummala PP, Kalpana R, Akhter MW, Karaalp IS, Wani OR et al. Maternal and fetal outcomes of subsequent pregnancies in women with peripartum cardiomyopathy. N Engl J Med. 2001; 344(21): 1567-71.

11. Faivre J, Verroust N, Ghiglione S, Mignon A. Cardiac diseases and pregnancy. Encycl Méd Chir (Elsevier Masson, Paris), Réanimation. 2009; $18: 215-22$

12. Goland S, Modi K, Bitar F, Janmohamed M, Mirocha JM, Czer LS et al. Clinical profile and predictors of complications in peripartum cardiomyopathy. J Card Fail 2009; 15:645-50

13. Groesdonk HV, Dinse-Lambracht A, Doblanzki W, Doblanzki U, Galm C, Muth CM. Unrecognized peripartum cardiomyopathy: case series and comprehensive review of the literature. Appl Cardiopulm Pathophysiol 2009;13:237-42

14. Jourdain P, Lefèvre G, Oddoze C, Sapin V, Dievart F, Jondeau G et al. NT-proBNP en pratique «De la biologie à la clinique». Ann Biol Clin $2009 ; 67$ (3) : 255-71

15. Katibi I. Peripartum cardiomyopathy in Nigeria. Hesp Med. 2003; 64(4):249.

16. Khemiri K, Jenayah AA, Boudaya F, Hamdi A, Meskhi S, Sfar E et al. Profil épidémiologique des femmes enceintes cardiaques dans le centre de maternité de Tunis: expérience du service A. Pan Afr Med J 2015; $21: 140$.

17. Klein LL, Galan HL. Cardiac disease in pregnancy. Obstet Gynecol Clin North Am 2004; 31: 429-59. 
18. Ladouceur M, Barre E, Radojevic J, Cohen S, Legendre A, Iserin L. Cardiopathie congénitale et grossesse. Consensus cardio. 2011; $72: 1$ 12.

19. Lutomski JE, Morrison JJ, Greene RA, Lydon-Rochelle MT. Maternal morbidity during hospitalization for delivery. Obstetrics \& Gynecology, 2011; 117(3): 596-602.

20. Machihude P, Yaovi A, Soodougoua B, Edem GA, Souleymane P, Borgatia A et al. Particularités de la cardiomyopathie du péripartum en Afrique: le cas du Togo sur une étude prospective de 41 cas au Centre Hospitalier et Universitaire Sylvanus Olympio de Lomé. Pan Afr Med J. 2014; $17: 245$.

21. McMurray JJ, Adamopoulos S, Anker SD, Auricchio A, Böhm M, Dickstein $\mathrm{K}$ et al. ESC Guidelines for the diagnosis and treatment of acute and chronic heart failure 2012: The Task Force for the Diagnosis and Treatment of Acute and Chronic Heart Failure 2012 of the European Society of Cardiology. Developed in collaboration with the Heart Failure Association (HFA) of the ESC. Eur Heart J. 2012 Jul;33(14):1787-847

22. Mielniczuk LM, Williams K, Davis DR, Tang AS, Lemery R, Green $\mathrm{MS}$ et al. Frequency of peripartum cardiomyopathy. Am J Cardiol 2006; 97: 1765-8.

23. Mongo Ngamami SF, Ellenga Mbolla BF, Nzaka-Sikou S, KoualaLanda C, Ikama SM, Gombet TR et al. Cardiomyopathie du péripartum: Aspects épidémiologiques, cliniques et pronostiques dans le service de cardiologie et médecine interne du CHU de Brazzaville (Congo). CAMES Sante. 2004 ; 2(1):69-73.

24. Ngouala GABB, Affangla DA, Leye M, Kane A. The prevalence of symptomatic infantile heart disease at Louga Regional Hospital, Senegal. CVJA $2015 ; 26$ (4): 1-5.

25. Ntusi NB, Badri M, Gumedze F, Sliwa K, Mayosi BM. PregnancyAssociated Heart Failure: A Comparison of Clinical Presentation and Outcome between Hypertensive Heart Failure of Pregnancy and Idiopathic Peripartum Cardiomyopathy. PLoS One. 2015 Aug 7;10(8):e0133466. doi: 10.1371/journal.pone.0133466. eCollection 2015.

26. Pearl W. Familial occurrence of peripartum cardiomyopathy. Am Heart J 1995; 129: 421-2.

27. Regitz-Zagrosek V, Lundqvist CB, Borghi C, Cifkova R, Ferreira R, Foidart JM et al. ESC Guidelines on the management of cardiovascular diseases during pregnancy. Eur Heart J. 2011;32(24):3147-97. doi: 10.1093/eurheartj/ehr218. 
28. Schellpfeffer MA, Gillespie KH, Rohan AM, Blackwell SP. A Review of Pregnancy-Related Maternal Mortality in Wisconsin, 2006-2010. WMJ. 2015 Oct;114(5):202-7.

29. Shah T, Ather S, Bavishi C, Bambhroliya A, Ma T, Bozkurt B. Peripartum cardiomyopathy: A contempary review. Methodist Debakey Cardiovasc J. 2013; 9(1):38-43.

30. Steer P. Heart disease in pregnancy. Women's Health Medicine 2005; 2(2):18-21.

31. Tara F, Vakilian F, Moosavi-Baigy F, Salehi M, Moghiman T. Prenatal and Cardiovascular Outcome in Pregnant Patients With Dyspnea. Res Cardiovasc Med. 2015; 4(2): e20950.

32. Van Mook WN, Peeters L. Severe cardiac disease in pregnancy, part II: impact of congenital and acquired cardiac diseases during pregnancy. Curr Opin Crit Care 2005; 11: 435-48.

33. Zabsonre P, Bamouni J, Fall FR, Dao B, Dyemkouma FX. Epidémiologie des insuffisances cardiaques du péripartum : A propos de 116 cas a Bobo-Dioulasso. Médecine d'Afrique Noire 2000; 47 (4): $187-90$. 